\title{
Bound on a diffuse flux of ultrahigh energy neutrinos in the Arkani-Hamed-Dimopoulos-Dvali model
}

\author{
M. O. Astashenkov* \\ Department of Physics, Lomonosov Moscow State University, 119991 Moscow, Russian Federation
}

A. V. Kisselev ${ }^{\dagger}$

A.A. Logunov Institute for High Energy Physics, NRC “Kurchatov Institute," 142281 Protvino, Russian Federation

(Received 9 April 2018; published 14 December 2018)

\begin{abstract}
The search for downward-going and Earth-skimming ultrahigh energy cosmic neutrinos by the Surface Detector array of the Pierre Auger Observatory (PAO) is analyzed in a framework of the Arkani-HamedDimopoulos-Dvali (ADD) model with $n$ large extra dimensions under the assumption that diffuse neutrino flux $d N_{\nu} / d E_{\nu}$ has the form $k E_{\nu}^{-2}$ in the energy range $10^{17}-2.5 \times 10^{19} \mathrm{eV}$. The upper bound on the flux normalization $k$ is obtained in the ADD model, taking into account that no neutrino events were found at the PAO. It is shown that for some values of $n$ and $(n+4)$-dimensional Planck scale $M_{D}$ our bound on $k$ is more stringent than that of the Pierre Auger Collaboration. The lower bound on $M_{D}$ as a function of $n$ is obtained.
\end{abstract}

DOI: 10.1103/PhysRevD.98.123009

\section{INTRODUCTION}

Ultrahigh energy (UHE) cosmic neutrinos play an important role in particle physics and astrophysics. They help us to determine the composition of UHE cosmic rays, as well as their origin. In particular, the detection of UHE neutrino candidates by the Pierre Auger Observatory (PAO) in coincidence with gravitational wave $(\mathrm{GW})$ events could constrain the position of the source of GW [1]. Measuring the scattering of UHE cosmic neutrinos off atmospheric nucleons can probe a new physics that could modify the neutrino-nucleon cross section at energies above $10^{17} \mathrm{eV}$. The first observation of high-energy astrophysical neutrinos was done by the IceCube Collaboration in 2014 [2]. It was found that the neutrino-nucleon cross section agrees with predictions in the range 6.3-980 $\mathrm{TeV}$ [3].

To detect neutrino events with energies above $10^{17} \mathrm{eV}$, more powerful cosmic ray facilities such as the PAO [4] and Telescope Array [5] are needed. Recently, the Pierre Auger Collaboration reported on searches for downward-going (DG) UHE neutrinos [6]. The DG incline air showers [7-9] are initiated by cosmic neutrinos moving with large zenith

\footnotetext{
*mixa.astash@yandex.ru

alexandre.kisselev@ihep.ru
}

Published by the American Physical Society under the terms of the Creative Commons Attribution 4.0 International license. Further distribution of this work must maintain attribution to the author(s) and the published article's title, journal citation, and DOI. Funded by SCOAP ${ }^{3}$. angle that interact in the atmosphere near the Surface Detector (SD) array of the PAO. Note that the background from hadronic showers is very small at $E_{\nu}>10^{17} \mathrm{eV}$ and negligible above $10^{19} \mathrm{eV}$ [10]. The data were collected by the SD in the zenith angle bins $60^{\circ}-75^{\circ}$ and $75^{\circ}-90^{\circ}$ for a period that is equivalent of 6.4 years of a complete PAO SD working continuously.

The PAO also searched for Earth-skimming (ES) air showers $[11,12]$ induced by upward tau neutrinos at zenith angles $90^{\circ}-95^{\circ}$ that interact in the Earth producing tau leptons. In their turn, the tau leptons escape the Earth and initiate showers close to the SD.

No neutrino candidates were found. Assuming the diffuse flux of UHE neutrinos to be

$$
\frac{d N}{d E_{\nu}}=k E_{\nu}^{-2}
$$

in the energy range $1.0 \times 10^{17}-2.5 \times 10^{19} \mathrm{eV}$, the $90 \%$ C.L. single-flavor upper limit to the diffuse flux of UHE neutrinos was obtained by the Pierre Auger Collaboration

$$
k<6.4 \times 10^{-9} \mathrm{GeV} \mathrm{cm}^{-2} \mathrm{~s}^{-1} \mathrm{sr}^{-1} .
$$

This bound is approximately four times less than the Waxman-Bachall bound on cosmic neutrino production in optically thin sources [13]. Some cosmogenic neutrino models with a pure proton composition injected at the sources were rejected by the Auger limit (2). The maximum sensitivity of the SD of the PAO lies at the neutrino energies around $1 \mathrm{EeV}$ [6]. The IceCube fit of the 
diffuse single-flavor astrophysical neutrino flux [14], if extrapolated to $1 \mathrm{EeV}$, would give $E_{\nu}^{2} d N / d E_{\nu}=$ $0.3 \times 10^{-9} \mathrm{GeV} \mathrm{cm}^{-2} \mathrm{~s}^{-1} \mathrm{sr}^{-1}$.

The calculations of the exposure of the SD array of the $\mathrm{PAO}$ were done under assumption that neutrino-nucleon collisions in the atmosphere are described by the Standard Model (SM) interactions [in charged current (CC) and neutral current (NC) channels].

The goal of the present paper is to estimate the singleflavor bound on the diffuse flux of UHE cosmic neutrinos in the model with extra dimensions. Namely, the ArkaniHamed-Dimopoulos-Dvali (ADD) model [15] with $n$ extra flat spatial dimensions is considered. We assume that neutrino energy spectrum is of the form $E_{\nu}^{-2}(1)$ in the range $10^{17}-2.5 \times 10^{19} \mathrm{eV}$.

As we show below (see Fig. 3 in Sec. III), possible ED effects are small in a range of sensitivity of the detector IceCube. That is why in what follows we consider UHE neutrinos with $E_{\nu} \geq 10^{17} \mathrm{eV}$.

\section{SPACE-TIME WITH LARGE EXTRA DIMENSIONS (THE ADD MODEL)}

Let us briefly remind readers of the main features of the ADD model. The large extra dimensions scenario was postulated in Refs. [15]. Its metric looks like

$$
d s^{2}=g_{\mu \nu}(x) d x^{\mu} d x^{\nu}+\eta_{a b} d y^{a} d y^{b},
$$

where $\mu, \nu=0,1,2,3, a, b=1, \ldots n$, and $\eta_{a b}=$ $(-1, \ldots,-1)$. All $n$ extra dimensions are compactified with a size $R_{c}$.

There is a hierarchy relation between the reduced fundamental gravity scale in $D=4+n$ dimensions, $M_{D}$, and the reduced Planck mass, $\bar{M}_{\mathrm{Pl}}=M_{\mathrm{Pl}} / \sqrt{8 \pi}$,

$$
\bar{M}_{\mathrm{Pl}}^{2}=V_{n} M_{D}^{2+n}
$$

where $V_{n}$ is a volume of the compactified dimensions. $V_{n}=\left(2 \pi R_{c}\right)^{n}$, if the extra dimensions are of a toroidal form. In order for $M_{D}$ to be of order 1 or a few $\mathrm{TeV}$, the radius of the extra dimensions should be large. The compactification scale $R_{c}^{-1}$ ranges from $10^{-3} \mathrm{eV}$ to $10 \mathrm{MeV}$ as $n$ runs from 2 to 6 .

All SM gauge and matter fields are assumed to be confined to a three-dimensional brane embedded into a $(3+n)$-dimensional space, while the gravity lives in all $D$-dimensional space-time called bulk.

In linearized gravity we present the $D$-dimensional metric $G_{A B}$ in the form $(A, B=0,1, \ldots, 3+n)$

$$
G_{A B}(x, y)=\eta_{A B}+\frac{2}{M_{D}^{1+n / 2}} h_{A B}(x, y) .
$$

Performing the Kaluza-Klein (KK) mode expansion of the gravitational field $h_{A B}(x, y)$, we obtain the graviton interaction Lagrangian density

$$
\mathcal{L}_{\text {int }}(x)=-\frac{1}{\bar{M}_{\mathrm{Pl}}} T^{\mu \nu}(x) \sum_{n=0}^{\infty} h_{\mu \nu}^{(n)}(x),
$$

where $n$ labels the KK excitation level, and $T^{\mu \nu}(x)$ is the energy-momentum tensor of the matter on the brane. The masses of the KK graviton modes $h_{\mu \nu}^{(n)}$ are

$$
m_{n}=\frac{\sqrt{n_{a} n^{a}}}{R_{c}}, \quad n_{a}=\left(n_{1}, n_{2} \ldots n_{n}\right) .
$$

So, a mass splitting is $\Delta m \sim R_{c}^{-1}$ and we have the almost continuous spectrum of the gravitons.

One can see from (6) that the coupling of both massless and massive graviton is universal and very small $\left(\sim 1 / \bar{M}_{\mathrm{Pl}}\right)$. Nevertheless, all cross sections with real and virtual production of the massive $\mathrm{KK}$ gravitons are defined by the gravity scale $M_{D}$, but not by $\bar{M}_{\mathrm{Pl}}$.

For $n=1$ and $M_{D} \sim 1 \mathrm{TeV}$, it follows from (4) that $R_{c} \sim 10^{13} \mathrm{~cm}$, implying deviation from Newtonian gravity over solar system distances. So the case $n=1$ is completely excluded.

For $n \geq 2$, limits on the fundamental Planck scale $M_{D}$ in the context of the ADD model [15] were computed by the CMS [16-18] and ATLAS [19-21] Collaborations. In particular, in [17] a search for new physics in final states containing a photon and missing transverse momentum at $\sqrt{s}=13 \mathrm{TeV}$ was presented using data corresponding to an integrated luminosity $\mathcal{L}$ of $35.9 \mathrm{fb}^{-1}$. Values of the scale $M_{D}$ up to $2.85-2.90 \mathrm{TeV}$ were excluded for between two and six extra dimensions. The most stringent limits on $M_{D}$ are obtained in [18], which vary from $9.9 \mathrm{TeV}$ for $n=2$ to $5.3 \mathrm{TeV}$ for $n=6$ at $95 \%$ C.L. These constraints were obtained in a search of events with one or more energetic jets and large missing transverse momentum recorded at $\sqrt{s}=13 \mathrm{TeV}$ and $\mathcal{L}=35.9 \mathrm{fb}^{-1}$. Recently, the results in terms of lower limits on $M_{D}$ were also reported by the ATLAS Collaboration [21] from a search for new phenomena in events with energetic jet and large missing transverse momentum at $\sqrt{s}=13 \mathrm{TeV}$ and $\mathcal{L}=36.1 \mathrm{fb}^{-1}$. Values of $M_{D}$ below $7.7 \mathrm{TeV}$ for $n=2$ and below $4.8 \mathrm{TeV}$ for $n=6$ are excluded at $95 \%$ C.L.

There are a number of cosmological and astrophysical constraints on the scale $M_{D}$ for $n=2$, 3. The most restrictive limits on $M_{D}$ come from the effect of $\mathrm{KK}$ graviton emission on cooling of supernovae and neutronstar excess heat [22-27]. If large extra dimensions exist, KK gravitons would be emitted from the supernova core after collapse, compete with neutrino cooling, and shorten the observable signal. This argument has led to the strong bound $M_{D}>31 \mathrm{TeV}$ for $n=2$ and $M_{D}>$ 2.75 for $n=3$ [27]. Some constraint also follows from a KK contribution to the cosmic diffuse radiation [28]. Note, however, that all these limits rely on a number of assumptions. 


\section{NEUTRINO-NUCLEON CROSS SECTIONS}

We intend to consider ultrahigh energies of cosmic neutrino, $E_{\nu}>10^{17} \mathrm{eV}$. It corresponds to a large center-of-mass energy of the neutrino-proton collision, $\sqrt{s} \gtrsim 14 \mathrm{TeV}$. Thus, we are in a trans-Planckian region $\sqrt{s} \gg M_{D}$. At transPlanckian energies the scattering is described by classical physics $[29,30]$, if the impact parameter $b$ is larger than the $D$-dimensional Schwarzschild radius $R_{S}$ [31],

$$
R_{S}(s)=\frac{1}{M_{D}}\left[\frac{2^{n} \pi^{\frac{n-3}{2}} \Gamma\left(\frac{n+3}{2}\right)}{n+2} \frac{\sqrt{s}}{M_{D}}\right]^{\frac{1}{n+1}} .
$$

The trans-Planckian regime corresponds to the conditions

$$
\sqrt{s} \gg M_{D}, \quad \theta \sim\left(R_{S} / b\right)^{n+1},
$$

where $\theta$ is the scattering angle [29].

In the eikonal approximation [32], which is valid at small momentum transfer $(-t \ll s)$, the leading part of the scattering amplitude is obtained by summation of all ladder diagrams with graviton exchange in the $t$-channel $[29,30]$. The tree-level exchange of the $D$-dimensional graviton gives the following Born amplitude,

$$
\begin{aligned}
A_{\mathrm{Born}}(t) & =\frac{s^{2}}{M_{D}^{n+2}} \int \frac{d^{n} q_{n}}{t-q_{n}^{2}} \\
& =\pi^{n / 2} \Gamma(1-n / 2)\left(\frac{-t}{M_{D}^{2}}\right)^{n / 2-1}\left(\frac{s}{M_{D}^{2}}\right)^{2},
\end{aligned}
$$

where $q_{n}$ is the momentum transfer in the extra dimensions. Summing all loop diagrams leads to the eikonal formula

$$
A_{\mathrm{eik}}(s, t)=-2 i s \int d^{2} b e^{i q b}\left[e^{i \chi(b)}-1\right],
$$

with the eikonal phase $\left(q^{2}=-t\right)$

$$
\chi(b)=\frac{1}{2 s} \int \frac{d^{2} q}{(2 \pi)^{2}} e^{-i q b} A_{\mathrm{Born}}\left(q^{2}\right) .
$$

It has been calculated in $[29,30]$ (see also [33]) to be

$$
\chi(b)=\left(\frac{b_{c}}{b}\right)^{n}
$$

where

$$
b_{c}=\left[\frac{(4 \pi)^{n / 2-1} s \Gamma(n / 2)}{2 M_{D}^{n+2}}\right]^{1 / n} .
$$

As a result, the final expression of the eikonal amplitude (11) is given by

$$
A_{\mathrm{eik}}(s, t)=4 \pi s b_{c}^{2} F_{n}\left(b_{c} q\right),
$$

where the function $F_{n}(y)$ is defined as

$$
F_{n}(y)=-i \int_{0}^{\infty} d z z J_{0}(z y)\left[e^{i z^{-n}}-1\right] .
$$

The eikonal representation of the scattering amplitude is a good approximation, provided $b>R_{S}[29,30]$.

At UHEs the neutrino interacts essentially with the quarks (antiquarks) and gluons inside the nucleon. Let us define a fraction of the neutrino energy transferred to the nucleon

$$
y=\frac{E_{\nu}-E_{\nu}^{\prime}}{E_{\nu}}=\frac{Q^{2}}{x S},
$$

where $Q^{2}=-q^{2}, E_{\nu}\left(E_{\nu}^{\prime}\right)$ is the initial (final) energy of the neutrino, and $x$ is the fraction of nucleon momentum carried by parton $i(i=q, \bar{q}, g)$. Taking into account the above-mentioned formulas, we get the differential neutrinonucleon cross section

$$
\frac{d^{2} \sigma}{d x d y}=\pi s \sum_{i} x f_{i}\left(x, \mu^{2}\right) b_{c}^{4}(\hat{s})\left|F_{n}\left(b_{c} Q\right)\right|^{2},
$$

where $\hat{s}=x s$, and $Q=\sqrt{y \hat{s}}$. The quantities $f_{i}\left(x, \mu^{2}\right)$ are the parton distribution functions (PDFs). Following Ref. [33], we put $\mu^{2}=Q^{2}$. We use the CT14 set for the PDFs [34]. In order to calculate total cross sections, we integrate (18) in the region $Q_{0}^{2}<Q^{2}<R_{S}^{-2}$ [33]. As in [33], we put $Q_{0}^{2}=0.01 m_{W}^{2}$, where $m_{W}$ is the W-boson mass.

As it was mentioned above, the eikonal approximation can be used if $Q^{2}<R_{S}^{-2}\left(b>R_{S}\right)$. In the rest of the integration region $s \geq Q^{2}>R_{S}^{-2}$, which corresponds to the region $b<R_{S}$ in the impact parameter space, one expects that the neutrino and a parton inside the nucleon will form a black hole. In such a case, the cross section can be estimated as $[35,36]$

$$
\sigma_{\nu N \rightarrow \mathrm{BH}}(s)=\pi \sum_{i} \int_{\left(M_{\mathrm{bh}}^{\min }\right)^{2} / s}^{1} d x f_{i}\left(x, \bar{\mu}^{2}\right) R_{S}^{2}(\hat{s}),
$$

where $M_{\mathrm{bh}}^{\min }$ is the minimal value of the black hole. We put $\bar{\mu}^{2}=x s$. The dependence of $\sigma_{\nu N \rightarrow \mathrm{BH}}$ on the choice of $\bar{\mu}^{2}$ and $M_{\mathrm{bh}}^{\min }$ is discussed in [37-39]. For chosen $n, M_{D}$, we take $M_{\mathrm{bh}}^{\min }$ to be equal to the $95 \%$ C.L. lower limit on $M_{\mathrm{bh}}$ for the same $n$ and $M_{D}$ obtained by the CMS Collaboration [40]. One can conclude from Fig. 6 in [40] and numerical estimations of $R_{S}$ (8) that $M_{\mathrm{bh}}^{\min } \gg R_{S}^{-1}$ for all $E_{\nu}$, if $2 \leq n \leq 6$, and $2 \mathrm{TeV}<M_{D}<6 \mathrm{TeV}$.

The black hole production by cosmic rays was studied in a number of papers (see, for an example, [35], [37-39,41]).

As for the SM neutrino interaction, we adopt the neutrino-nucleon cross sections in [42], since the Pierre Auger Collaboration [6] has obtained limit (2) with the use of these SM cross sections. 

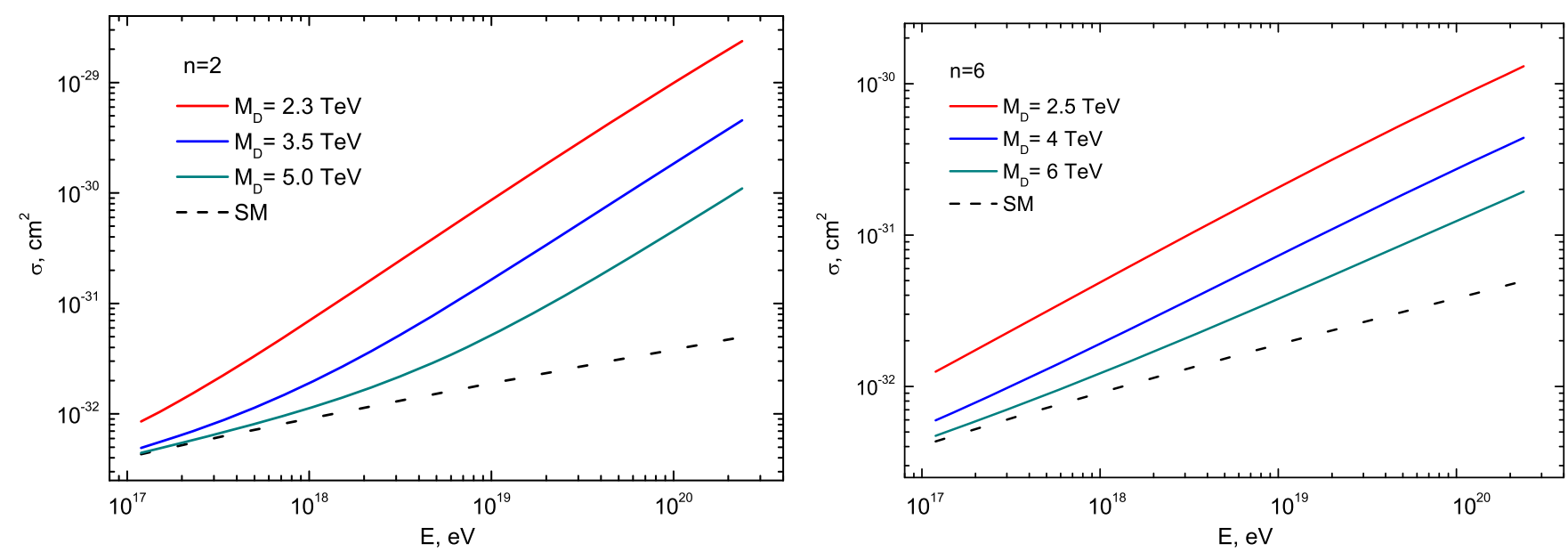

FIG. 1. Left panel: the neutrino total cross sections for $n=2$ and $M_{D}=2.3,3.5,5.0 \mathrm{TeV}$ (solid lines). Right panel: the same as on the left panel, but for $n=6$ and $M_{D}=2.5,4.0,6.0 \mathrm{TeV}$. For comparison, the neutrino CC total cross section is shown by the dashed lines.
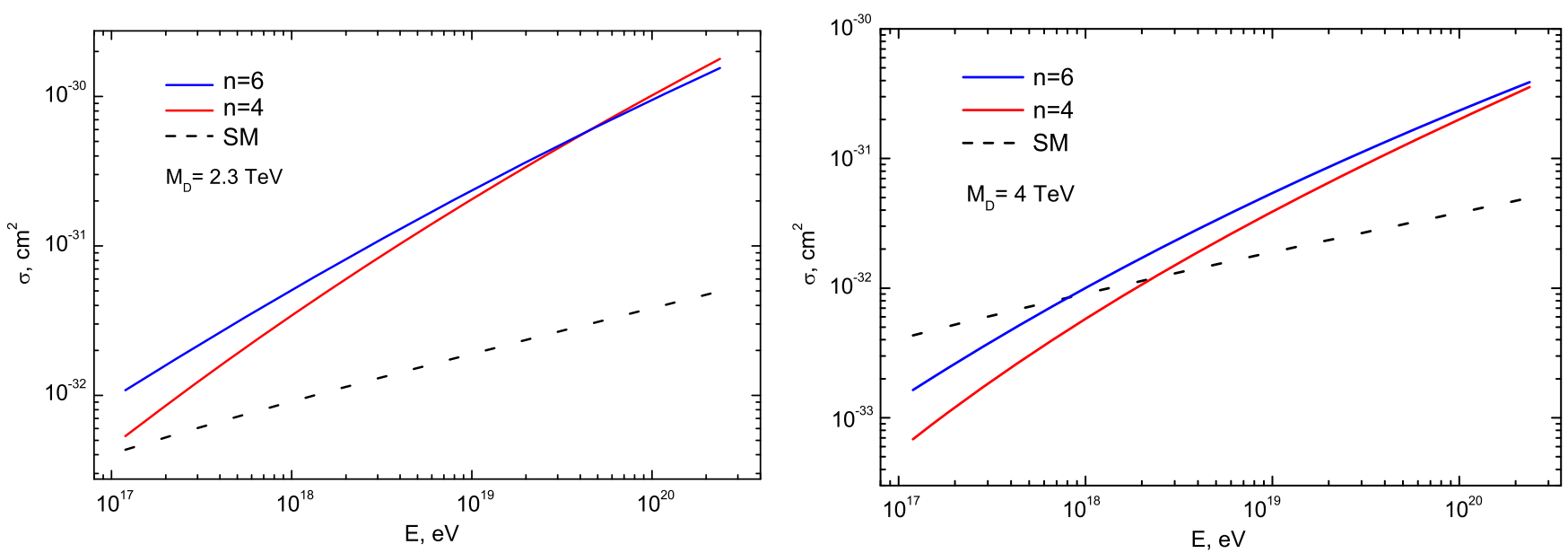

FIG. 2. Left panel: the neutrino cross sections in the ADD model for $M_{D}=2.3 \mathrm{TeV}$ and $n=4,6$ (solid lines, no SM contribution is included). The dashed line: the neutrino CC total cross section. Right panel: the same as on the left panel, but for $M_{D}=4$.

The total cross sections as functions of the $D$-dimensional mass scale $M_{D}$ and number of the extra dimensions $n$ are shown in Figs. 1 and 2. Let us note that at $E_{\nu}>10^{19} \mathrm{eV}$ the cross section $\sigma_{\nu N \rightarrow \mathrm{BH}}$ rises with $n$, while the eikonal cross section decreases. The combined effects of these two factors is that the difference of the cross sections for $n=4$ and $n=6$ tends to 0 as $E_{\nu}$ grows (see Fig. 2).

In Fig. 3 the neutrino-nucleon cross section is presented in a range of sensitivity of the detector IceCube. As one can see, possible ED effects are small in this energy region. That is why in what follows we consider UHE neutrinos with $E_{\nu} \geq 10^{17} \mathrm{eV}$.

Our calculations of the cross sections are not an end in itself but will enable us to estimate exposures for both DG and ES neutrino events at the SD array of the PAO in the ADD model and thus to put limits on the diffuse singleflavor flux of UHE neutrinos.

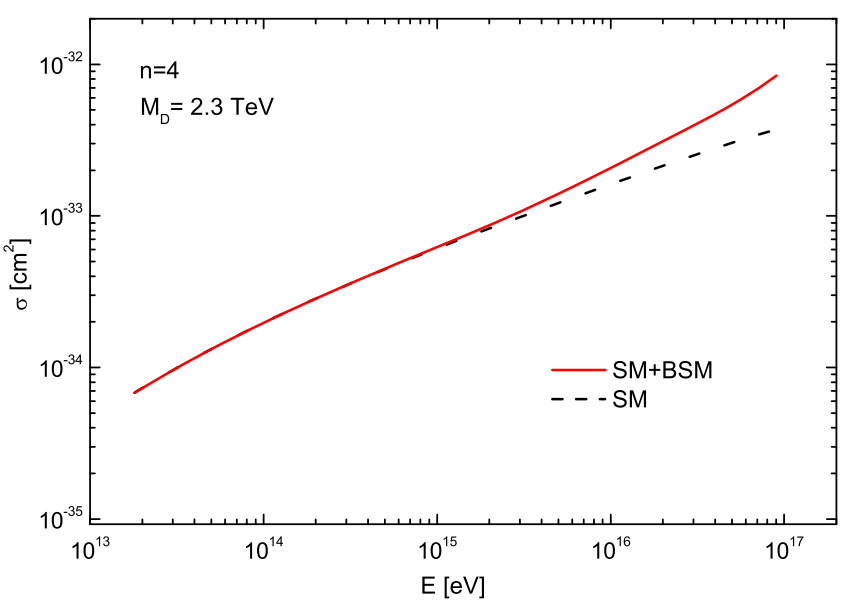

FIG. 3. The neutrino-nucleon cross sections for $M_{D}=2.3 \mathrm{TeV}$, $n=4$ in the energy range $10^{13} \leq E_{\nu} \leq 10^{17} \mathrm{eV}$. 


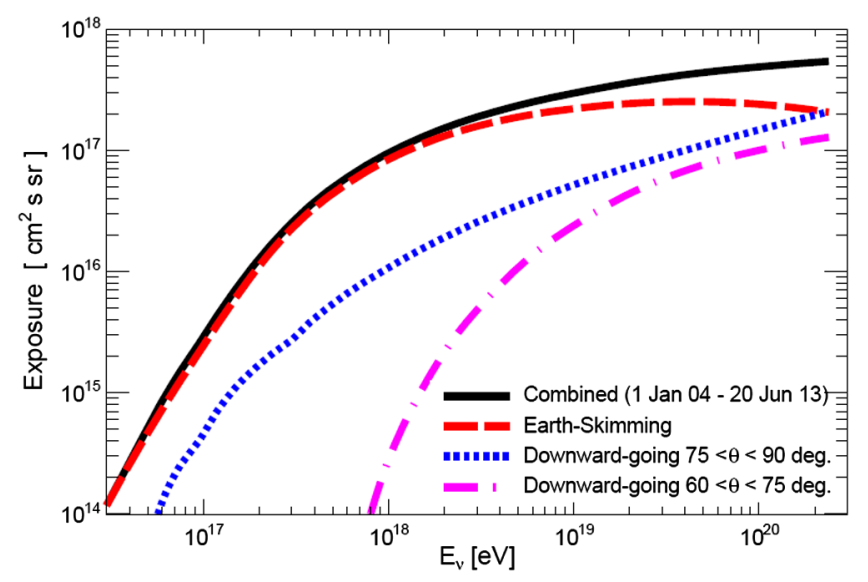

FIG. 4. The combined exposure of the SD array of the PAO (1 January 2004-20 June 2013) as a function of the neutrino energy. The individual exposures are also shown (Fig. 3 from Ref. [6]).

\section{LIMITS ON DIFFUSE FLUX OF UHE NEUTRINOS IN THE ADD MODEL}

In Refs. [10,37] the following functional dependence of the DG event rate on the new physics cross section $\sigma_{\mathrm{NP}}$ was proposed for quasihorizontal UHE neutrino events

$$
\mathcal{E}_{\mathrm{BSM}}^{\mathrm{DG}}\left(E_{\nu}\right)=\mathcal{E}_{\mathrm{SM}}^{\mathrm{DG}}\left(E_{\nu}\right) \frac{\sigma_{\mathrm{SM}}^{\mathrm{eff}}\left(E_{\nu}\right)+\sigma_{\mathrm{NP}}\left(E_{\nu}\right)}{\sigma_{\mathrm{SM}}^{\mathrm{eff}}\left(E_{\nu}\right)}
$$

where $\mathcal{E}_{\mathrm{BSM}}^{\mathrm{DG}}\left(\mathcal{E}_{\mathrm{SM}}^{\mathrm{DG}}\right)$ is the exposure of the $\mathrm{SD}$ of the $\mathrm{PAO}$ with (without) account of the new interaction. In addition, instead of $\sigma_{\mathrm{CC}}$, an effective SM cross section $\sigma_{\mathrm{SM}}^{\text {eff }}$ is introduced in (20),

$$
\sigma_{\mathrm{SM}}^{\mathrm{eff}}=\sigma_{\mathrm{CC}} \sum_{i=e, \mu, \tau} m_{\mathrm{CC}}^{i}+3 \sigma_{\mathrm{NC}} m_{\mathrm{NC}}+\sigma_{\mathrm{CC}} m_{\mathrm{mount}}
$$

Here $m_{\mathrm{CC}}^{i}$ and $m_{\mathrm{NC}}$ are relative mass apertures for CC and $\mathrm{NC}$ interactions of the DG neutrinos at the PAO. The mass

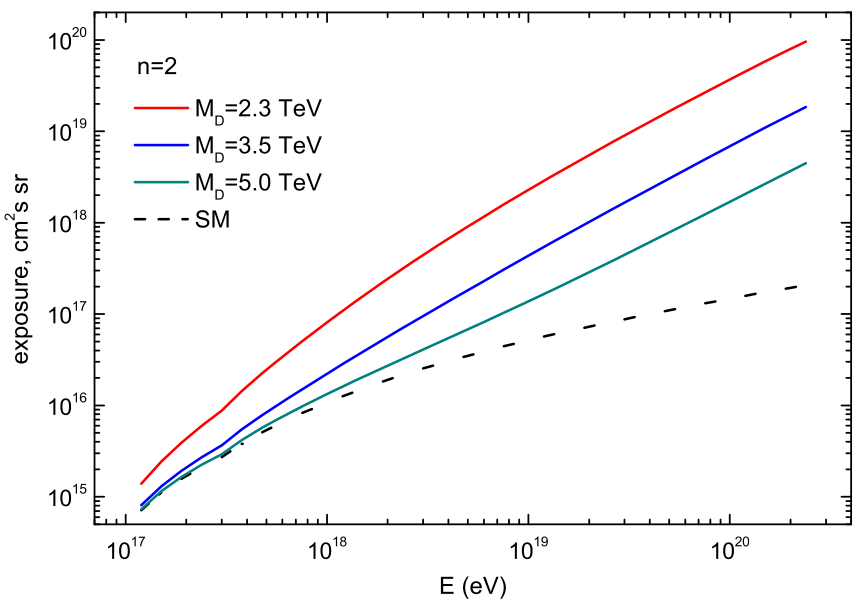

aperture $m_{\text {mount }}$ corresponds to the CC interaction of a $\tau$ neutrino within the mountains around the PAO. The relative mass apertures as functions of the neutrino energy were calculated using the data in Table I of Ref. [43]. Note that $\sum_{i=e, \mu, \tau} m_{\mathrm{CC}}^{i}+3 m_{\mathrm{NC}}+m_{\text {mount }}=1$.

Equation (20) follows simply from the fact that at fixed $E_{\nu}$ an exposure of a DG event is proportional to the neutrino-nucleon cross section.

In contrast to the DG neutrino exposure, the exposure of the ES tau neutrinos decreases with the rise of the neutrino total cross section [10,37],

$$
\mathcal{E}_{\mathrm{BSM}}^{\mathrm{ES}}\left(E_{\nu}\right)=\mathcal{E}_{\mathrm{SM}}^{\mathrm{ES}}\left(E_{\nu}\right) \frac{\sigma_{\mathrm{CC}}^{2}\left(E_{\nu}\right)}{\left[\sigma_{\mathrm{CC}}\left(E_{\nu}\right)+\sigma_{\mathrm{NP}}\left(E_{\nu}\right)\right]^{2}} .
$$

This formula was obtained using the fact that at Auger energies the neutrino interaction length $L_{\text {int }}^{\nu}$ satisfies the condition $L_{\text {int }}^{\nu} \gg L^{\tau}$, where $L^{\tau}$ is the maximal path length for a detectable $\tau$. Note that this inequality is valid when the cross section enhancement is significant but not as large as the typical hadronic cross section.

The formulas (20) and (22) allowed us to calculate exposures of the SD of the PAO for the period 1 January 2004-20 June 2013 expected in the ADD model. The PAO data on the exposures for the SM neutrino interactions in the region from $\log \left(E_{\nu} / \mathrm{eV}\right)=17$ to 20.5 were used (see Fig. 4 taken from Ref. [6]). The results of our calculations are presented in Figs. 5 and 6.

Note that previously, analogous calculations were done for the PAO exposure in the Randall-Sundrum scenario [44].

We assume that the astrophysical flux arrives isotropically from all directions, and neutrino flavor composition is $\nu_{e}: \nu_{\mu}: \nu_{\tau}=1: 1: 1$. Following Pierre Auger Collaboration, we also assume that the flux is described by a power law of the form (1). Then the upper limit on the value of $k$ can be estimated as [6]

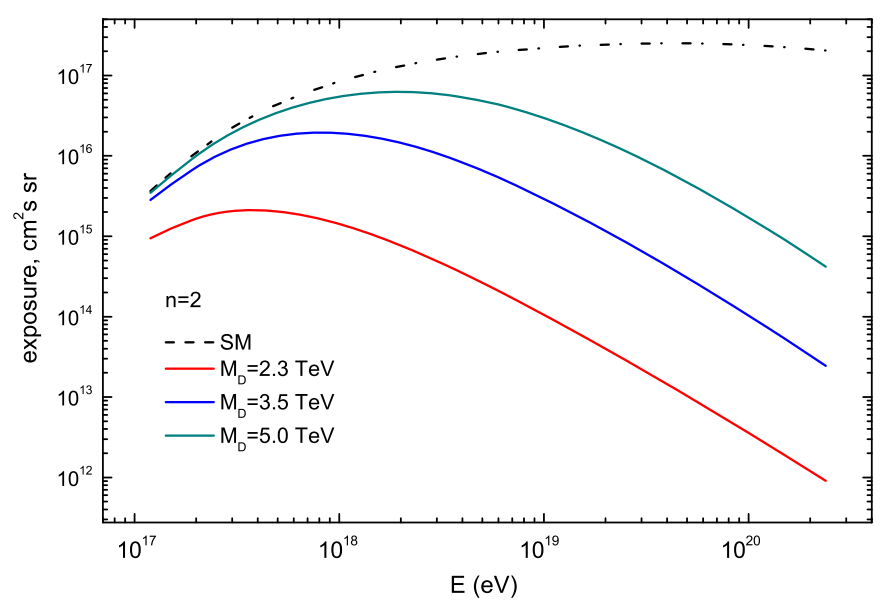

FIG. 5. Left panel: the expected exposures of the SD array of the PAO for the DG neutrinos with zenith angle $75^{\circ}<\theta<90^{\circ}$ in the ADD model. Right panel: the expected exposures of the SD array of the PAO for the ES neutrinos in the ADD model. 

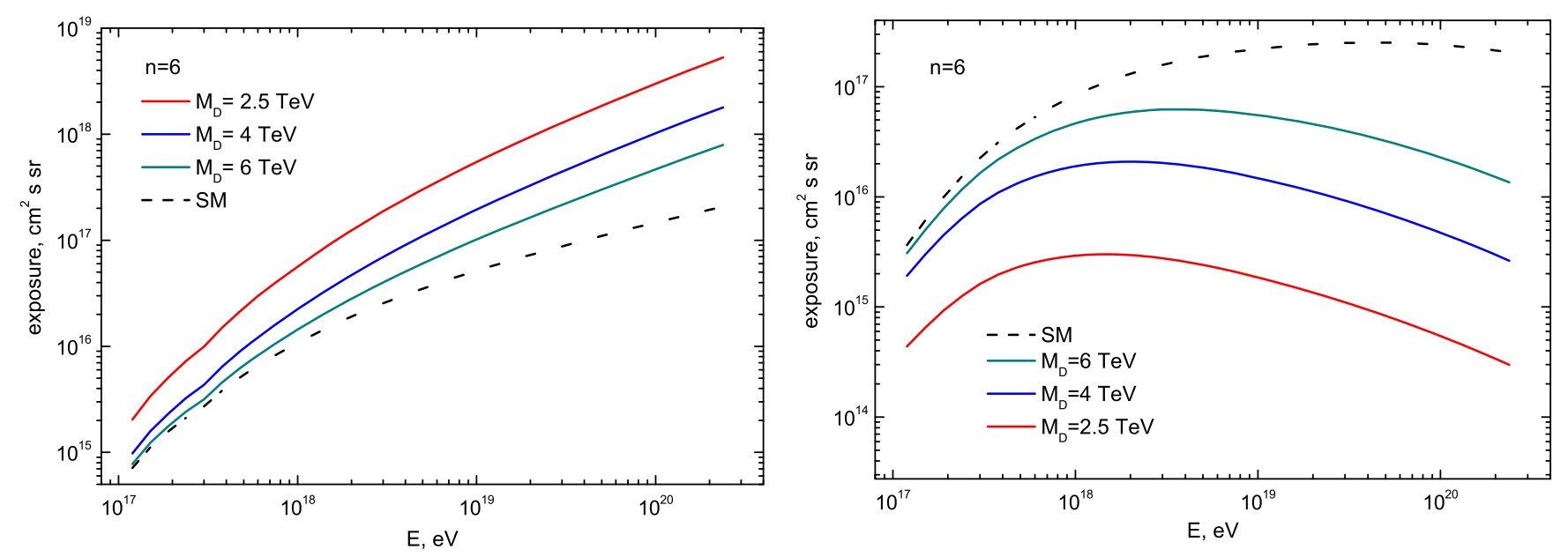

FIG. 6. The same as in Fig. 5, but for $n=6$.

$$
k=\frac{N_{\mathrm{up}}}{\int E_{\nu}^{-2} \mathcal{E}_{\mathrm{tot}}\left(E_{\nu}\right) d E_{\nu}},
$$

where $N_{\text {up }}$ is an actual value of the upper limit on the signal events, which depends on the number of the observed events and total exposure

$$
\mathcal{E}_{\mathrm{tot}}=\mathcal{E}_{\mathrm{BSM}}^{\mathrm{DG}}+\mathcal{E}_{\mathrm{BSM}}^{\mathrm{ES}}
$$

see Eqs. (20) and (22). The value of $N_{\text {up }}$ depends on the number of observed events, expected background events, and confidence level required (90\% C.L. is assumed). Since the PAO sees no events, we put $N_{\text {up }}=2.39$, assuming a number of expected background events to be 0 [6].

As one can see in Fig. 1, in the ADD model the cross sections rise more rapidly with the neutrino energy than the SM cross sections. As a result, the exposure for the DG

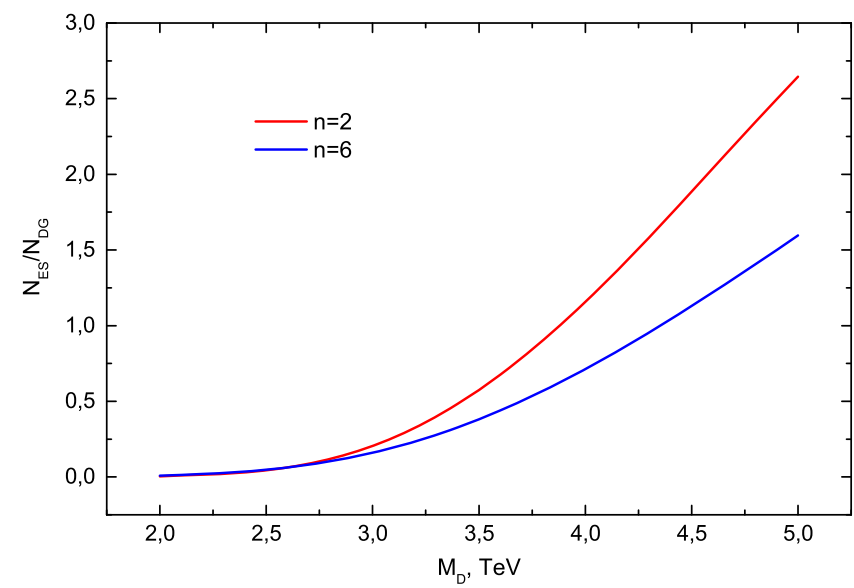

FIG. 7. The expected ratio of the ES neutrinos to the DG neutrinos with zenith angle $75^{\circ}<\theta<90^{\circ}$ at the SD array of the PAO as a function of the gravity scale $M_{D}$ for two values of $n$. events, $\mathcal{E}_{\mathrm{BSM}}^{\mathrm{DG}}(20)$, rises, while the exposure for the ES events, $\mathcal{E}_{\mathrm{BSM}}^{\mathrm{ES}}(22)$, decreases as $E_{\nu}$ grows (see Figs. 5 and 6). The expected ratio of the ES neutrinos to the DG neutrinos with zenith angle $75^{\circ}<\theta<90^{\circ}$ is shown in Fig. 7 as a function of the gravity scale $M_{D}$. One can see that this ratio rapidly grows, as $M_{D}$ gets larger. It is a manifestation of the different dependence of the DG and ES exposures on the neutrino cross section.

As a result, for some values of $n$ and $M_{D}$, the total expected exposure in the ADD model (24) can be larger than the Auger exposure calculated on the assumption that the neutrino-nucleon scattering is defined by the SM interactions only. Correspondingly, the upper bound on $k$ defined by Eq. (23) can be even stronger than the bound obtained by the Pierre Auger Collaboration (2). It is demonstrated by Figs. 8 and 9. It enables us to obtain a lower bound on $M_{D}$ as a function of $n$ presented in Fig. 10.

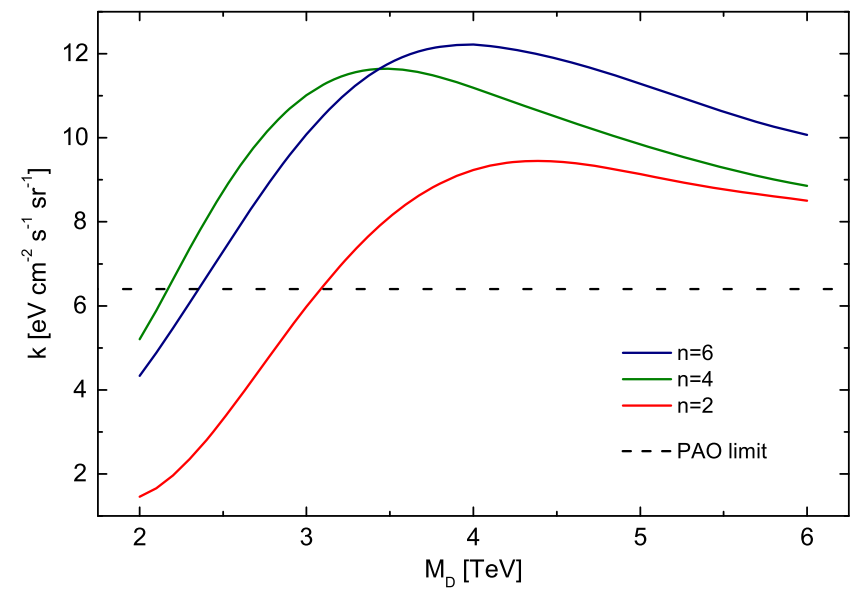

FIG. 8. The upper bound on the value of $k$ as a function of D-dimensional Planck scale $M_{D}$ for different values of $n=2$. The dashed line is the PAO upper limit [6]. 


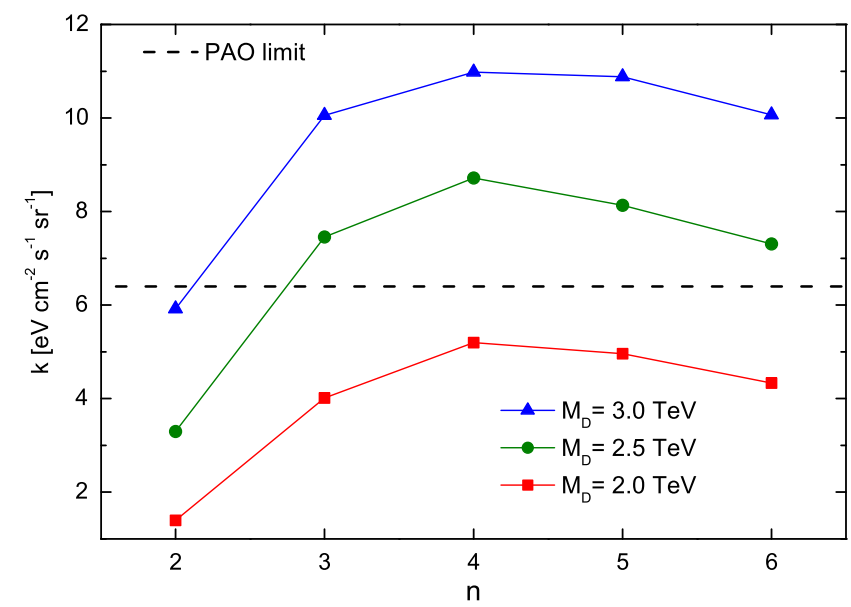

FIG. 9. The upper bound on the value of $k$ as a function of number of extra dimensions $n$ for different values of $M_{D} \mathrm{TeV}$. The dashed line is the PAO upper limit [6].

\section{CONCLUSIONS AND DISCUSSIONS}

In the present paper the neutrino cross sections in the ADD model [15] with $n$ extra dimensions (EDs) were calculated (Figs. 1 and 2). It is shown that ED effects are small in a range of sensitivity of the neutrino detector IceCube (Fig. 3). That is why we considered UHE neutrinos with energies $E_{\nu} \gtrsim 10^{17} \mathrm{eV}$.

Using the exposure of the PAO for the period equivalent of 6.4 years of the complete PAO SD array working continuously, the exposures for neutrino induced events were calculated both for downward-going and Earthskimming UHE neutrinos. Their dependence on $n$ and fundamental gravity scale $M_{D}$ was obtained (Figs. 5 and 6).

Our main goal was to calculate a single-flavor upper limit on the diffuse neutrino flux in the ADD model. Following [6], we assumed that the differential neutrino

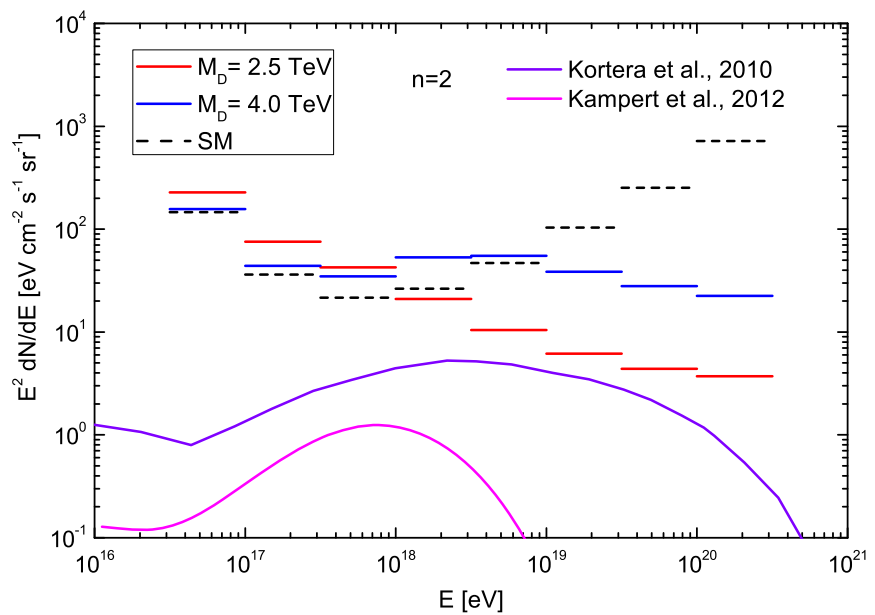

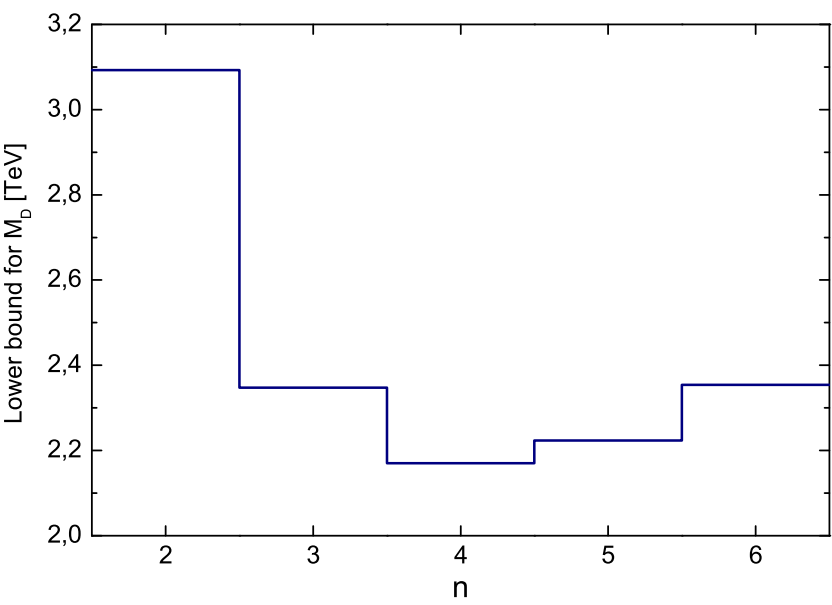

FIG. 10. The lower bound on the fundamental scale of the ADD model $M_{D}$ as a function of the number of extra dimensions $n$.

flux has the form $k E_{\nu}^{-2}$. We have found that for some values of $n$ and $M_{D}$, our bound on the flux normalization $k$ is stronger than that of the Pierre Auger Collaboration (2). As one can see in Fig. 8, it takes place for $M_{D}<$ $3.09 \mathrm{TeV}(2.35 \mathrm{TeV})$, if $n=2$ (6). Figure 9 shows us that our value of $k$ overestimates the PAO limit for all $n$, provided $M_{D}>3 \mathrm{TeV}$.

It can be understood as follows. Recall that the upper limit on the neutrino diffuse flux (1) is given by formula (23). In the ADD model the neutrino-nucleon cross sections grow with energy faster than the SM cross sections (Figs. 1 and 2). Correspondingly, the expected exposure for the DG neutrino events (20) gets higher. On the contrary, for the ES neutrino events $\mathcal{E}_{\mathrm{BSM}}^{\mathrm{ES}}$ decreases as neutrino energy grows (Figs. 5 and 6). As a result, the total exposure $\mathcal{E}_{\text {tot }}$ (24) multiplied by $E_{\nu}^{-2}$ may be larger than that of the Pierre Auger Collaboration (Fig. 4), provided that an

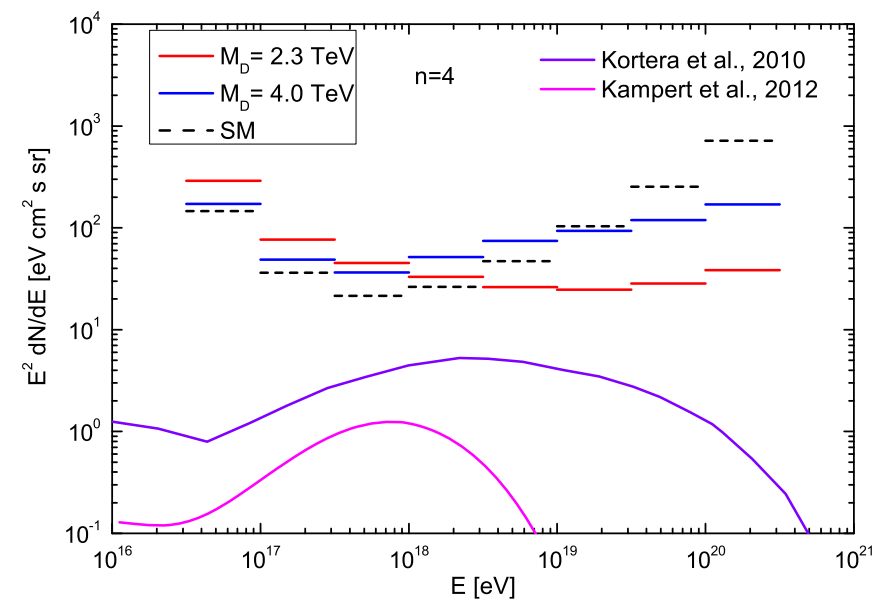

FIG. 11. Left panel: the upper bound on the diffuse neutrino flux in bins for two values of $M_{D}$ and $n=2$ (solid lines) in comparison with the PAO bound (dashed lines) and predictions of two cosmogenic models $[45,46]$ (solid lines). Right panel: the same as on the left panel, but for $n=4$. 
integrated increase of $E_{\nu}^{-2} \mathcal{E}_{\mathrm{BSM}}^{\mathrm{DG}}\left(E_{\nu}\right)$ prevails over an integrated reduction of $E_{\nu}^{-2} \mathcal{E}_{\mathrm{BSM}}^{\mathrm{ES}}\left(E_{\nu}\right)$.

At fixed $n$, the upper limit on the flux normalization $k$ tends from above to the PAO limit, as $M_{D}$ grows (2) (see Fig. 8). For rather large $M_{D}$, our bound becomes weaker than the PAO bound for all $n$ (see Fig. 9). It enables us to put the lower bound on $M_{D}$ as a function of $n$ (Fig. 10).

We have also calculated an upper bound on the normalization of the diffuse flux of UHE neutrinos in bins (Fig. 11). Two curves in this figure are the cosmogenic fluxes obtained under the assumption that the composition of the primary flux is iron rich [45] or pure iron [46]. The SM prediction corresponds to Fig. 5 in Ref. [6]. As one can see, the ADD model does not constrain such kind of cosmogenic models. As for cosmogenic models that assume a pure proton primary flux [46,47], we did not compare our bounds with them, since they are already disfavored by the PAO limit (2) [6]. Moreover, the pure proton primary flux is excluded by PAO data on a mass composition of cosmic rays above $10^{17.2} \mathrm{eV}$, except for a small energy region around $10^{18.3} \mathrm{eV}$ [48].

\section{ACKNOWLEDGMENTS}

The authors are very indebted to J. Alvarez-Muñiz for sending us the data on the exposures of the SD array of the $\mathrm{PAO}$ and two cosmogenic fluxes.
[1] A. Aab et al. (Pierre Auger Collaboration), Phys. Rev. D 94, 122007 (2016).

[2] M. G. Aartsen et al. (IceCube Collaboration), Phys. Rev. Lett. 113, 101101 (2014).

[3] M. G. Aartsen et al. (IceCube Collaboration), Nature (London) 551, 596 (2017).

[4] J. Abraham et al. (Pierre Auger Collaboration), Nucl. Instrum. Methods Phys. Res., Sect. A 523, 50 (2004).

[5] T. Abu-Zayyad et al. (Telescope Array Collaboration), Nucl. Instrum. Methods Phys. Res., Sect. A 689, 87 (2012).

[6] A. Aab et al. (Pierre Auger Collaboration), Phys. Rev. D 91, 092008 (2015).

[7] V. S. Berezinsky and G. T. Zatsepin, Phys. Lett. 28B, 423 (1969); V. S. Berezinsky and A. Yu. Smirnov, Astrophys. Space Sci. 32, 461 (1975).

[8] K. S. Capelle, J. W. Cronin, G. Parente, and E. Zas, Astropart. Phys. 8, 321 (1998).

[9] E. Zas, New J. Phys. 7, 130 (2005).

[10] L. Anchordoqui, H. Goldberg, D. Góra, T. Paul, M. Roth, S. Sarkar, and L. L. Winders, Phys. Rev. D 82, 043001 (2010).

[11] X. Bertou, P. Billoir, O. Deligny, C. Lachaud, and A. Letessier-Selvon, Astropart. Phys. 17, 183 (2002).

[12] J. L. Feng, P. Fisher, F. Wilczek, and T. M. Yu, Phys. Rev. Lett. 88, 161102 (2002).

[13] E. Waxman and J. N. Bachall, Phys. Rev. D 64, 023002 (2001).

[14] M. G. Aartsen et al. (IceCube Collaboration), Phys. Rev. D 91, 022001 (2015).

[15] N. Arkani-Hamed, S. Dimopoulos, and G. Dvali, Phys. Lett. B 429, 263 (1998); I. Antoniadis, N. Arkani-Hamed, S. Dimopoulos, and G. Dvali, Phys. Lett. B 436, 257 (1998); N. Arkani-Hamed, S. Dimopoulos, and G. Dvali, Phys. Rev. D 59, 086004 (1999).

[16] A. M. Sirunyan et al. (CMS Collaboration), J. High Energy Phys. 10 (2017) 073.

[17] A. M. Sirunyan et al. (CMS Collaboration), arXiv:1810 .00196 .
[18] A. M. Sirunyan et al. (CMS Collaboration), Phys. Rev. D 98, 092001 (2018).

[19] M. Aaboud et al. (ATLAS Collaboration), J. High Energy Phys. 06 (2016) 059.

[20] M. Aaboud et al. (ATLAS Collaboration), Phys. Rev. D 94, 032005 (2016).

[21] M. Aaboud et al. (ATLAS Collaboration), J. High Energy Phys. 01 (2018) 126.

[22] S. Cullen and M. Perelstein, Phys. Rev. Lett. 83, 268 (1999).

[23] V. Barger, T. Han, C. Kao, and R.-J. Zhang, Phys. Lett. B 461, 34 (1999).

[24] C. Hanhart, D. R. Phillipsa, S. Reddy, and M. J. Savage, Nucl. Phys. B595, 335 (2001).

[25] S. Hannestad and G. Raffelt, Phys. Rev. Lett. 87, 051301 (2001).

[26] S. Hannestad and G. Raffelt, Phys. Rev. D 67, 125008 (2003); 69, 029901(E) (2004).

[27] S. Hannestad and G. Raffelt, Phys. Rev. Lett. 88, 071301 (2002).

[28] L. J. Hall Lawrence and D. Smith, Phys. Rev. D 60, 085008 (1999).

[29] G. F. Giudice, R. Rattazzi, and J. D. Wells, Nucl. Phys. B630, 293 (2002).

[30] R. Emparan, M. Masip, and R. Rattazzi, Phys. Rev. D 65, 064023 (2002).

[31] R. C. Myers and M. J. Perry, Ann. Phys. (N.Y.) 172, 304 (1986); P. C. Argyres, S. Dimopoulos, and J. March-Russel, Phys. Lett. B 441, 96 (1998).

[32] H. Cheng and T. T. Wu, Phys. Rev. Lett. 22, 666 (1969); H. Abarbanel and C. Itzykson, Phys. Rev. Lett. 23, 53 (1969); M. Levy and J. Sucher, Phys. Rev. 186, 1656 (1969).

[33] E. M. Sessolo and D. W. McKay, Phys. Lett. B 668, 396 (2008).

[34] S. Dulat, T.-J. Hou, J. Gao, M. Guzzi, J. Huston, P. Nadolsky, J. Pumplin, C. Schmidt, D. Stump, and C.-P. Yuan, Phys. Rev. D 93, 033006 (2016).

[35] J. L. Feng and A. D. Shapere, Phys. Rev. Lett. 88, 021303 (2001). 
[36] S. B. Giddings and S. Thomas, Phys. Rev. D 65, 056010 (2002).

[37] L. A. Anchordoque, J. L. Feng, H. Golgberg, and A. D. Shapere, Phys. Rev. D 65, 124027 (2002).

[38] L. A. Anchordoque, J. L. Feng, H. Golgberg, and A. D. Shapere, Phys. Rev. D 68, 104025 (2003).

[39] E.-J. Ahn, M. Ave, M. Cavaglià, and A. V. Olinto, Phys. Rev. D 68, 043004 (2003).

[40] A. M. Sirunyan et al. (CMS Collaboration), Phys. Lett. B 774, 279 (2017).

[41] A. Ringwald and H. Tu, Phys. Lett. B 525, 135 (2002).

[42] A. Cooper-Sarkar and S. Sarkar, J. High Energy Phys. 01 (2008) 075.
[43] P. Abreu et al. (Pierre Auger Collaboration), Phys. Rev. D 84, 122005 (2011).

[44] A. V. Kisselev and L. A. Shkalina, EPJ Web Conf. 125, 02014 (2016).

[45] K. Kotera, D. Allard, and A. V. Olinto, J. Cosmol. Astropart. Phys. 10 (2010) 013.

[46] K.-H. Kampert and M. Unger, Astropart. Phys. 35, 660 (2012).

[47] M. Ahlers, L. A. Anchordoqui, M. C. Gonzalez-Garcia, F. Halzen, and S. Sarkar, Astropart. Phys. 34, 106 (2010).

[48] A. Aab et al. (Pierre Auger Collaboration), Phys. Rev. D 96, 122003 (2017). 